\title{
On the validity of a numerical model predicting heat and mass transfer in porous square cavities with a bottom thermal and solute source: case of pollutants spreading and fuel leaks
}

\author{
Karim Ragui ${ }^{a}$, Abdelkader Boutra and Youb Khaled Benkahla \\ Laboratory of Transfer Phenomenon, University of Sciences and Technology Houari Boumediene, BP. 32 El Alia, \\ 16111 Bab Ezzouar, Algiers, Algeria
}

Received 8 December 2014, Accepted 14 November 2015

\begin{abstract}
The present work refers to the study of natural convection into a confined porous medium, driven by cooperating thermal and solutal buoyancy forces. The side walls are maintained at a uniform temperature and concentration, lower than that of a heat and solute source, which located at the center of the bottom wall, the rest of the horizontal walls are kept insulated. The physical model for the momentum conservation equation makes use of the Brinkman extension of the classical Darcy equation, the set of coupled equations is solved using the finite volume method and the SIMPLER algorithm. To account for the effects of the main parameters such the buoyancy ratio, the Lewis and porous thermal Rayleigh numbers, as well as the source length, heat and mass transfer characteristics are widely inspected and then, new powerful correlations are proposed, which predict within $\pm 1 \%$ the numerical results. Note that the validity of the used code was ascertained by comparing our results with experimental data and numerical ones already available in the literature.
\end{abstract}

Key words: Double-diffusive convection / square porous enclosure / thermal and solute source / finite volume method / heat and mass correlations

\section{Introduction}

Over the past four decades, double-diffusive natural convection analysis into a porous medium has been the subject of a very intense research activity, due to the importance of related industrial and contemporary technological applications such grain storage installation; geothermal energy resources; petroleum reservoirs; pollutant dispersion in aquifers; fibrous insulating materials, electrochemical processes, and some modes of assisted oil recuperation [1].

With both temperature and concentration gradients present to drive the fluid flow, an increased number of transport configurations was possible, with parallel or perpendicular gradients, and the body forces augmenting or opposing [2].

Several experimental [3-6] and numerical investigations [7-10] caused by horizontal thermal and solutal gradients have been done. For certain conditions, a multicellular flow was observed experimentally or predicted numerically. In contrast, a little attention was dedicated to the case of vertical gradients [11-14].

\footnotetext{
${ }^{a}$ Corresponding author: ragui-karim@live.fr
}

To predict the heat and mass rates into such configurations, some powerful correlations were proposed: in 1987, Trevisan and Bejan [15] projected the thermal and solutal transfer characteristics into a porous square as a function of pertinent parameters such: Lewis and porous thermal Rayleigh numbers; the cavity aspect ratio and the buoyancy ratio as well. In 1990, Lin et al. [16] proposed new Nusselt and Sherwood correlations as a function of the thermal Grashof number, and that for small values of the buoyancy ratio $(|N|<5)$. A few years later, Bennacer [17] suggested a general correlation for the mass transfer, which can be used in a wide range of porous thermal Rayleigh number, buoyancy ratio, and Lewis number as well.

In these works and many others, the problem of double-diffusive convection was commonly inspected using the entire wall length of the enclosure as a heat (and solute) source, what made these correlations only available using this condition. Thus, the main purpose of the current work is to complete these cases by investigating the double-diffusive natural convection within a cold (and less concentric) sides porous square, which including a heat and solute source located at the center of its bottom wall. This especial geometry is an interesting work 


\section{Nomenclature}

\begin{tabular}{|ll|}
\hline$C$ & Dimensional solute concentration \\
$D$ & Mass diffusivity $\left(\mathrm{m}^{2} \cdot \mathrm{s}^{-1}\right)$ \\
$D a$ & Darcy number, $=K / H^{2}$ \\
$G r_{T}$ & Thermal Grashof number, $=g \beta_{T} \Delta T H^{3} / \nu^{2}$ \\
$H$ & Cavity height $(\mathrm{m})$ \\
$K$ & Porous medium permeability $\left(\mathrm{m}^{2}\right)$ \\
$L e$ & Lewis number, $=S c / P r$ \\
$N$ & Buoyancy ratio, $=\beta_{C} \Delta C / \beta_{T} \Delta T$ \\
$N u$ & Mean Nusselt number \\
$p^{*}$ & Pressure (Pa) \\
$P$ & Dimensionless pressure \\
$P r$ & Prandtl number, $=\nu / \alpha$ \\
$R a_{T}$ & Thermal Rayleigh number, $=G r_{T} P r$ \\
$R a^{*}$ & Porous thermal Rayleigh number, $=R a_{T} D a$ \\
$S c$ & Schmidt number, $=\nu / D$ \\
$S h$ & Mean Sherwood number \\
$T$ & Dimensional temperature $(\mathrm{K})$ \\
$u, v$ & Velocity components $\left(\mathrm{m} . \mathrm{s}^{-1}\right)$ \\
$U, V$ & Dimensionless velocity components, $=u($ or $v) H / v$ \\
$w$ & Source length $(\mathrm{m})$ \\
$W$ & Dimensionless source length, $w / H$ \\
$x, y$ & Cartesian coordinates $(\mathrm{m})$ \\
$X, Y$ & Dimensionless Cartesian coordinates, $x($ or $y) / H$ \\
$G r e e k$ & symbols \\
$\alpha$ & Thermal diffusivity $\left(\mathrm{m}^{2} \cdot \mathrm{s}^{-1}\right)$ \\
$\beta_{C}$ & Solutal expansion coefficient \\
$\beta_{T}$ & Thermal expansion coefficient $\left(\mathrm{K}^{-1}\right)$ \\
$\varepsilon$ & Medium porosity \\
$\phi$ & Dimensionless concentration, $=\left(C-C^{-}\right) /\left(C^{+}-C^{-}\right)$ \\
$\theta$ & Dimensionless temperature, $=\left(T-T_{c}\right) /\left(T_{h}-T_{c}\right)$ \\
$\nu$ & Kinematic viscosity $\left(\mathrm{m}^{2} \cdot \mathrm{s}^{-1}\right)$ \\
$\Psi$ & Stream function \\
Subscripts & Cotd \\
\hline &
\end{tabular}

for many industrial applications, especially in predicting pollutants spreading and fuel leaks in the Refining and Petrochemical industry.

\section{Problem statement and mathematical formulation}

The studied configuration, shown in Figure 1, consists of a square porous cavity (of impermeable boundaries) saturated by a binary fluid. The side walls are subjected to uniform temperature and concentration lower than that of a heat and solute source located at the center of the bottom wall, when zero heat and mass fluxes

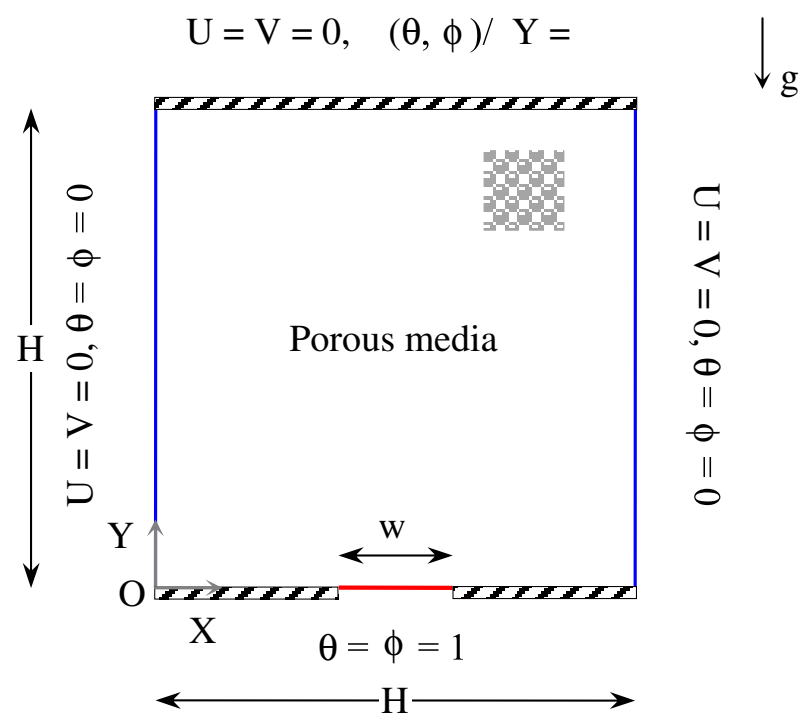

Fig. 1. Simulation domain with its boundary conditions.

are imposed at the rest parts of the horizontal walls. The binary fluid is assumed to be Newtonian and to satisfy the Boussinesq approximation; the flow is incompressible, laminar, bi-dimensional and in the steady state. On the other hand, the porous medium is supposed to be isotropic, homogeneous and in thermodynamic equilibrium with the fluid. The Soret and Dufour effects are assumed to be negligible.

The density variations upon temperature and concentration are described by the state equation:

$$
\rho(T, C)=\rho_{0}\left[1-\beta_{T}\left(T-T_{0}\right)-\beta_{\mathrm{C}}\left(C-C_{0}\right)\right]
$$

where $\beta_{T}$ and $\beta_{C}$ are thermal and solutal expansion coefficients.

$$
\beta_{T}=-\frac{1}{\rho_{0}}\left[\frac{\partial \rho}{\partial T}\right]_{C, p} \text { and } \beta_{C}=-\frac{1}{\rho_{0}}\left[\frac{\partial \rho}{\partial C}\right]_{T, p}
$$

The dimensionless conservation equations, describing transport phenomena in the square, can be written as:

$$
\begin{gathered}
\frac{\partial U}{\partial X}+\frac{\partial V}{\partial Y}=0 \\
\frac{1}{\varepsilon^{2}}\left(U \frac{\partial U}{\partial X}+V \frac{\partial U}{\partial Y}\right)=-\frac{\partial P}{\partial X}-\frac{1}{D a} U+\left(\frac{\partial U^{2}}{\partial X^{2}}+\frac{\partial U^{2}}{\partial Y^{2}}\right) \\
\frac{1}{\varepsilon^{2}}\left(U \frac{\partial V}{\partial X}+V \frac{\partial V}{\partial Y}\right)=-\frac{\partial P}{\partial Y}-\frac{1}{D a} V \\
+\left(\frac{\partial V^{2}}{\partial X^{2}}+\frac{\partial V^{2}}{\partial Y^{2}}\right)+G r_{T}(\theta+N \varphi) \\
U \frac{\partial \theta}{\partial X}+V \frac{\partial \theta}{\partial Y}=\frac{1}{P r}\left[\frac{\partial^{2} \theta}{\partial X^{2}}+\frac{\partial^{2} \theta}{\partial Y^{2}}\right] \\
U \frac{\partial \varphi}{\partial X}+V \frac{\partial \varphi}{\partial Y}=\frac{1}{S c}\left[\frac{\partial^{2} \varphi}{\partial X^{2}}+\frac{\partial^{2} \varphi}{\partial Y^{2}}\right]
\end{gathered}
$$


where $D a$ is the Darcy number, $G r_{T}\left(=R a_{T} / P r\right)$ is the thermal Grashof number, $N$ is the buoyancy ratio, $\mathrm{Pr}$ and $S c$ are the Prandtl and Schmidt numbers.

The appropriate boundary conditions used to solve the above system (3 to 7 ) can be resumed as follows:

$$
\begin{aligned}
& X=0 \quad 0<Y<1 \quad U=V=0 \quad \theta=\phi=0 \\
& X=1 \quad 0<Y<1 \quad U=V=0 \quad \theta=\phi=0 \\
& Y=0 \quad 0<x<\frac{1-W}{2} \quad U=V=0 \\
& \left.\frac{\partial \theta}{\partial Y}\right|_{Y=0}=\left.\frac{\partial \varphi}{\partial Y}\right|_{Y=0}=0 \\
& \frac{1-W}{2}<x<\frac{1+W}{2} \quad U=V=0 \quad \theta=\phi=1 \\
& \frac{1+W}{2}<x<1 \quad U=V=\left.0 \quad \frac{\partial \theta}{\partial Y}\right|_{Y=0}=\left.\frac{\partial \varphi}{\partial Y}\right|_{Y=0}=0 \\
& Y=1 \quad 0<x<1 \quad U=V=0 \\
& \left.\frac{\partial \theta}{\partial Y}\right|_{Y=1}=\left.\frac{\partial \varphi}{\partial Y}\right|_{Y=1}=0
\end{aligned}
$$

Noted that average heat and mass fluxes across the cavity' side walls, as well as the bottom source, can be expressed in a dimensionless form by the Nusselt and Sherwood numbers such as:

$$
\begin{array}{r}
\left|N u_{\text {side wall }}\right|=\left.\int_{0}^{1}\left(\frac{\partial \theta}{\partial X}\right)\right|_{\text {wall }} \mathrm{d} Y \\
\left|N u_{\text {bottom source }}\right|=\left.\int_{\frac{1-W}{2}}^{\frac{1+W}{2}}\left(\frac{\partial \theta}{\partial Y}\right)\right|_{\text {source }} \mathrm{d} X \\
\left|S h_{\text {side wall }}\right|=\left.\int_{0}^{1}\left(\frac{\partial \varphi}{\partial X}\right)\right|_{\text {wall }} \mathrm{d} Y ; \\
\left|S h_{\text {bottom source }}\right|=\left.\int_{\frac{1-W}{2}}^{\frac{1+W}{2}}\left(\frac{\partial \varphi}{\partial Y}\right)\right|_{\text {source }} \mathrm{d} X
\end{array}
$$

\section{Numerical procedure and code validation}

The governing conservation equations are discretized in space using the finite volume approach, when the convection-diffusion terms are treated with a Power-Law scheme. The resulting algebraic equations, with the associated boundary conditions, are then solved using the line by line method. As the momentum equation is formulated in terms of the primitive variables $(U, V$ and $P)$, the iterative procedure includes a pressure correction calculation method, namely SIMPLER [18] to solve the pressurevelocity coupling. Compared to other velocity-pressure coupling approaches such SIMPLE and SIMPLEC, the
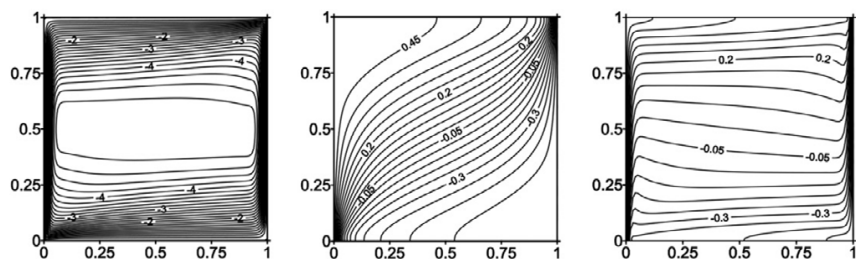

Hadidi et al. [19]
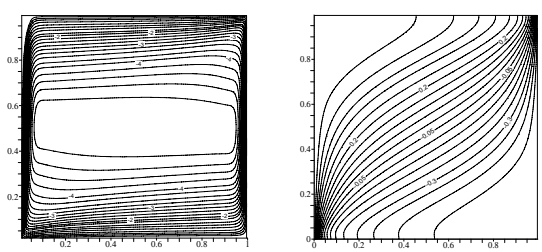

(b)

(a)

Present predictions

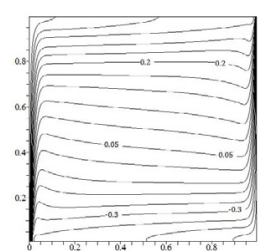

(c)
Fig. 2. Streamlines (a), isotherms (b) and iso-concentration lines (c), $R a=10^{6}, \operatorname{Pr}=7, D a=10^{-4}, L e=10, N=10$.

Table 1. Average Nusselt and Sherwood numbers obtained with our computer code and those of Hadidi et al. [19]. $D a=$ $10^{-4}, L e=10, \operatorname{Pr}=7, \operatorname{Ra}=10^{6}$.

\begin{tabular}{ccccc}
\hline \multirow{2}{*}{$N$} & \multicolumn{2}{c}{ Hadidi et al. [19] } & \multicolumn{2}{c}{ Present work } \\
\cline { 2 - 5 } & $N u$ & $S h$ & $N u$ & $S h$ \\
\hline 0 & 2.83 & 10.25 & 2.79 & 10.29 \\
10 & 3.95 & 26.30 & 3.91 & 26.33 \\
15 & 4.57 & 29.75 & 4.56 & 29.81 \\
\hline
\end{tabular}

adopted approach is proved to be faster (about 30 to $50 \%$ fewer iterations).

The convergence criterion for the temperature, the concentration, the pressure, and the velocity as well, is given as:

$$
\frac{\sum_{j=1}^{m} \sum_{i=1}^{n}\left|\xi_{i, j}^{t+1}-\xi_{i, j}^{t}\right|}{\sum_{j=1}^{m} \sum_{i=1}^{n}\left|\xi_{i, j}^{t+1}\right|} \leq 10^{-5}
$$

where both $m$ and $n$ are the numbers of grid points in $X$ and $Y$-directions, respectively; $\xi$ is any of the computed field variables and $t$ is the iteration number.

The performance of the using code via the doublediffusive natural convection problem into a porous medium is accomplished by comparing predictions with other numerical results and experimental data, and by verifying the grid independence of the present results.

First, the present results are consistent with previous computations namely those of Hadidi et al. [19] which deal with double-diffusive natural convection into a square enclosure formed by two different porous media. By taking into account the same hypotheses, Figure 2 shows the developed streamlines, isotherms and iso-concentration plots into the square. Tables 1 and 2 illustrate the comparison of mean Nusselt and Sherwood numbers computed with various values of the buoyancy ratio $N$ and the porous thermal Rayleigh number $R a^{*}$, respectively. 
Table 2. Average Nusselt and Sherwood numbers obtained with our computer code and those of Hadidi et al. [19]. $D a=$ $10^{-4}, L e=10, N=10, \operatorname{Pr}=7$.

\begin{tabular}{ccccc}
\hline \multirow{2}{*}{$R a^{*}$} & \multicolumn{2}{c}{ Hadidi et al. [19] } & \multicolumn{2}{c}{ Present work } \\
\cline { 2 - 5 } & $N u$ & $S h$ & $N u$ & $S h$ \\
\hline 1 & 1.02 & 3.28 & 1.04 & 3.30 \\
100 & 3.95 & 26.30 & 3.91 & 26.33 \\
\hline
\end{tabular}

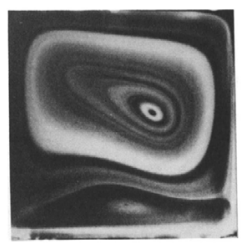

(a)

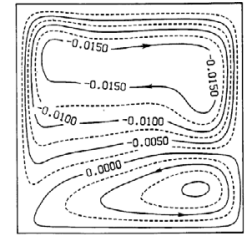

(b)
Weaver and Viskanta [20]

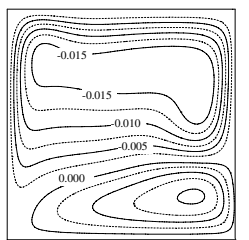

Present prediction

Fig. 3. Ethanol/Nitrogen binary fluid in a square cavity, (a) $G r=1.15710^{6}, N=-2.335, \operatorname{Pr}=0.802, S c=0.555$. (b) $G r=1.12110^{6}, N=-2.328, \operatorname{Pr}=0.802, S c=0.557$.

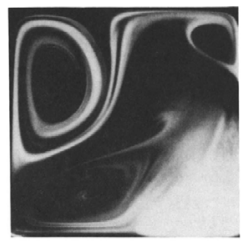

(a)

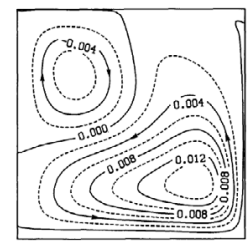

(b)
Weaver and Viskanta [20]

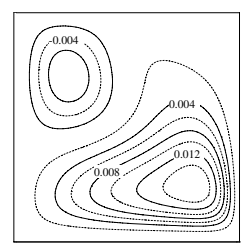

Present prediction
Fig. 4. Ethanol/Helium binary fluid in a square cavity, (a) $G r=2.71010^{4}, N=-5.475, \operatorname{Pr}=0.531, S c=1.574$. (b) $G r=2.68910^{4}, N=-5.411, P r=0.533, S c=1.588$.

As we can see, the present results and those of Hadidi et al. [19] are in excellent agreement with a maximum discrepancy of about $2 \%$.

Then, to check the numerical code validity with experimental results, those obtained by Weaver and Viskanta [20] for an Ethanol/Nitrogen and Ethanol/Helium binary fluids have been selected. Figures 3 and 4 display the comparison between the experimental data and both the numerical Weaver and Viskanta predictions and the present ones in term of velocity contours. Once again, the numerical results show a good qualitative concordance with the experimental data and a great agreement with the numerical Weaver and Viskanta predictions.

In order to determine a proper grid for the numerical simulations, a grid independence study is conducted. Several mesh distributions ranging from $91^{2}$ to $151^{2}$ are tested; the mean Nusselt and Sherwood numbers of the porous square, previously shown in Figure 1, are presented in Figure 5 as a function of the above uniform grids. Through the latter, a $121^{2}$ uniform grid is found to be adequate for a grid independent solution. However, a

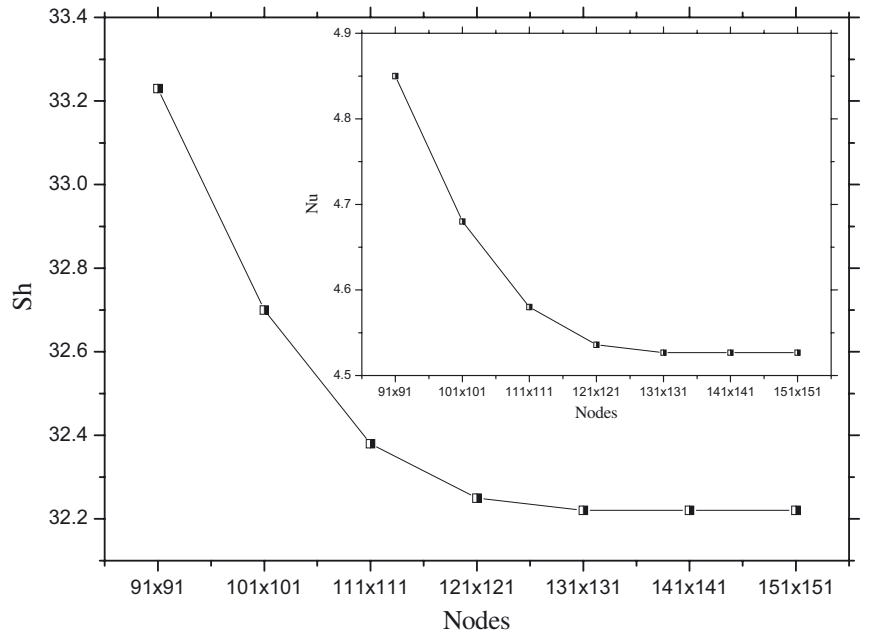

Fig. 5. Sherwood and Nusselt numbers of the square porous enclosure for different uniform grids, $D a=10^{-3}, L e=30$, $N=10, \operatorname{Pr}=10, R a^{*}=100, W=0.60$.

fine structured mesh of $131^{2}$ will be used to avoid roundoff error for all other calculations in our investigation.

\section{Results and discussion}

In this paper, the predictions are performed for various values of the Lewis number $(10 \leq L e \leq 300)$, the buoyancy ratio $(0 \leq N \leq 50)$, and the source length $(0.20 \leq W \leq 0.80)$. Both; the Darcy and Prandtl numbers are fixed at $10^{-3}$ and 10 , respectively. As the square cavity is formed by a solid matrix, our results are presented using a porous thermal Rayleigh number $\left(100 \leq R a^{*} \leq 2000\right)$ rather than the thermal one $\left(R a_{T}\right)$.

\subsection{Impact of Lewis number and buoyancy ratio}

Starting our investigation by a fixed source length equal to 0.60, Figure 6 displays the streamlines, the isotherms and the iso-concentration plots, according to Lewis number and that, for a buoyancy ratio and a porous thermal Rayleigh number equal 10 and 100, respectively.

For these patterns, two symmetrical counter-rotating cells are formed into the porous square, the fluid flows up in the centre on the enclosure and down all along the side walls at a relatively high speed with a low Lewis number, compared to the fluid speed with a high one.

The corresponding isotherms are found to be nearly the same, and barely changed, demonstrating a dominated-conduction heat transfer regime, unlike the isoconcentration plots where the latter becomes more distorted by increasing the Lewis number. Then, mass boundary layers are created all along the active walls and become thinner with increasing Lewis values. Also a solutal plume appears in the center of the enclosure indicating then, the domination of the diffusion mechanism.

For more clarification, the hydrodynamic, thermal and solutal behaviors are summarized in Figure 7 where the 

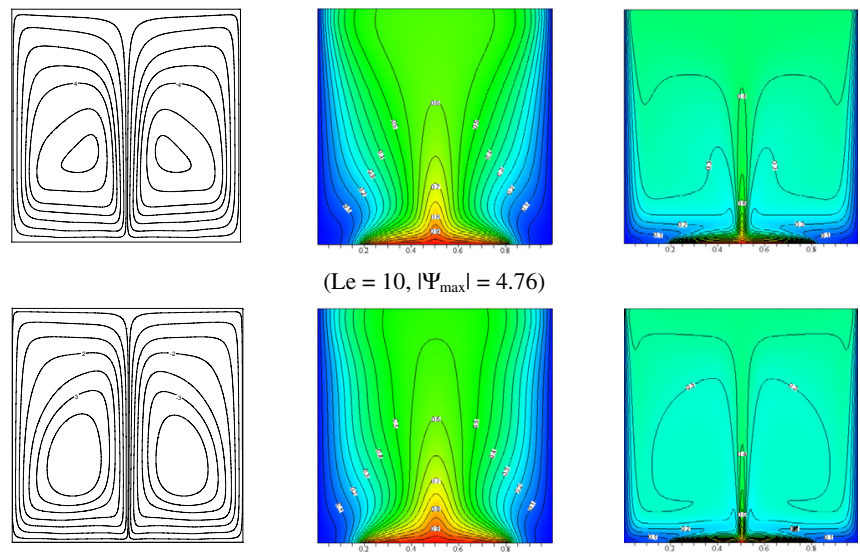

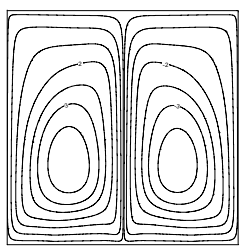

Streamlines
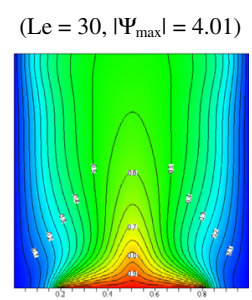

$\left(\mathrm{Le}=50,\left|\Psi_{\max }\right|=3.79\right)$

Isotherms

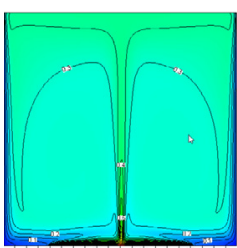

Iso-concentrations
Fig. 6. Impact of Lewis number, $N=10, R a^{*}=100$ $W=0.60$.

vertical velocity component, the temperature and the concentration profiles, along the horizontal mid-plane of the porous enclosure, are presented. In fact, the examination of the magnitude of $V$-velocity at various Lewis numbers confirms the results previously obtained from analysing the streamlines. The vertical velocity profiles verify the existence of clockwise and anti-clockwise circulating cells inside the porous square. Besides, the decrease in the velocity magnitude with increasing Lewis number is a clear indication of a weak buoyant flow at high values of the latter. In the same way, the mass transfer mechanism is expected to be more pronounced by increasing Lewis number, as illustrated via the $\phi$ profiles, whilst the conduction is responsible for the heat transfer as shown by the $\theta$ profiles.

Regarding the buoyancy ratio effect, Figure 8 shows the fluid dynamic, thermal and solutal behaviors, in terms of streamlines isotherms and iso-concentrations, for various values of $N$ (the Lewis number is fixed now at 10). Once again two symmetrical counter-rotating cells are formed into the porous square. Its absolute streamfunction value is an increasing function of the buoyancy ratio as the fluid motion becomes stronger. The corresponding isotherms are more pronounced by increasing values of $N$, indicating the transition from a pseudoconductive regime (at $N=1$ ) to a primary-convective one (see for instance $N=30$ ). As to the concentration field which is very affected by increasing buoyancy ratio, thin mass boundary layers are created all around the active walls, denoting large concentration gradients along these surfaces. The thickness of the latter (mass bound-
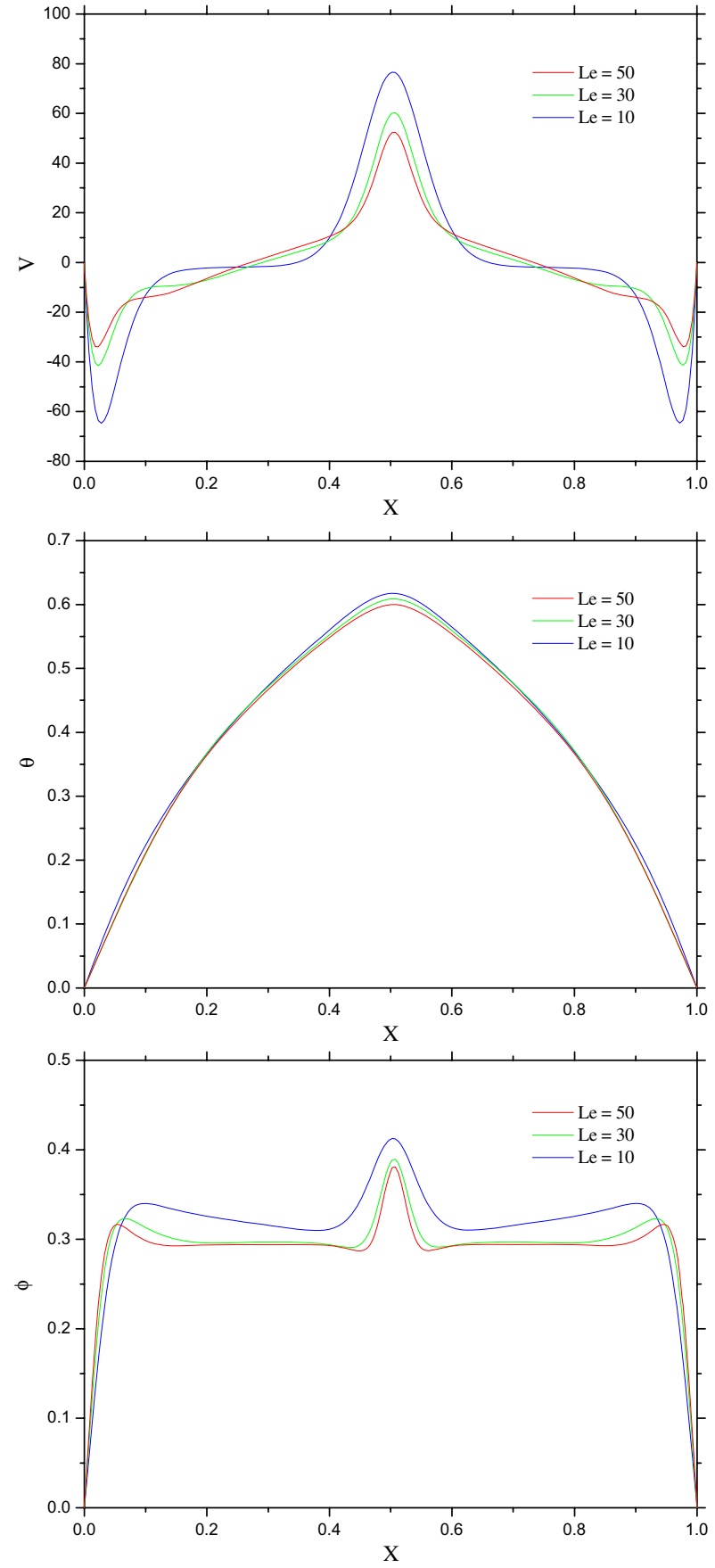

Fig. 7. $V$-Velocity, $\theta$ and $\phi$ profiles along the horizontal midplane of the porous square for various values of Lewis number. $N=10, R a^{*}=100, W=0.60$.

ary layer), is a decreasing function of enhanced values of $N$ which may explain the domination of the diffusion mechanism compared to the convection one.

Referred to Figure 9, the velocity magnitude and $\phi$ profiles depend not only to the Lewis values but to the buoyancy ratio ones as well. As we can see, an important buoyant flow and mass transfer is also produced using a high value of buoyancy ratio compared to a lower one. Note, at the end, that the effect of the buoyancy 

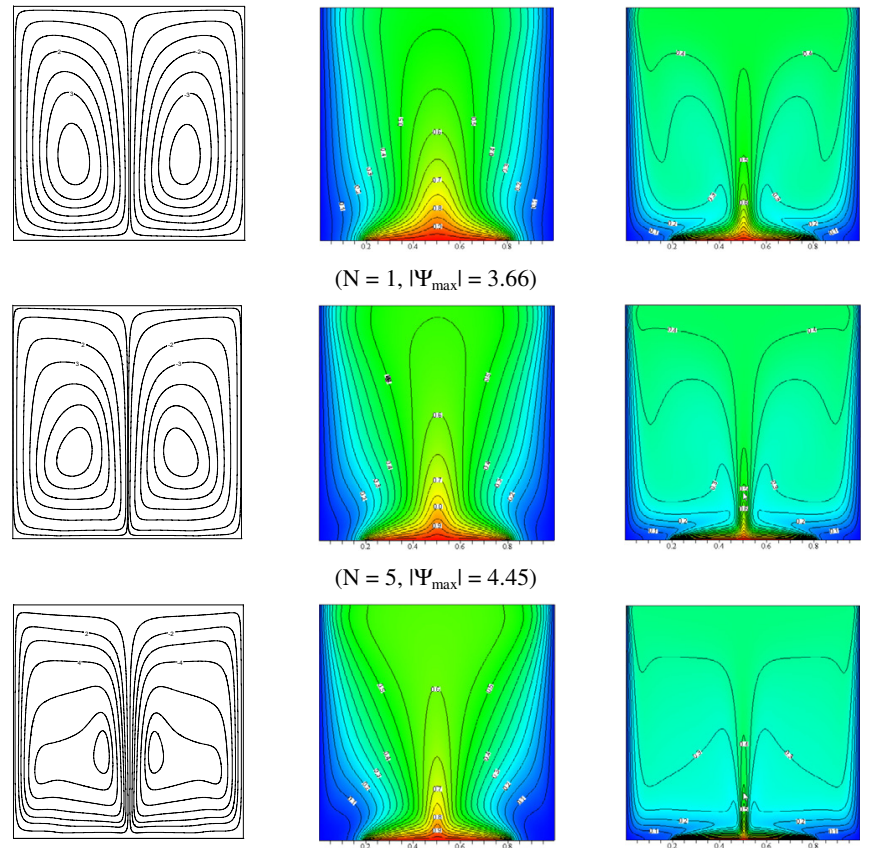

$\left(\mathrm{N}=20,\left|\Psi_{\max }\right|=5.72\right)$
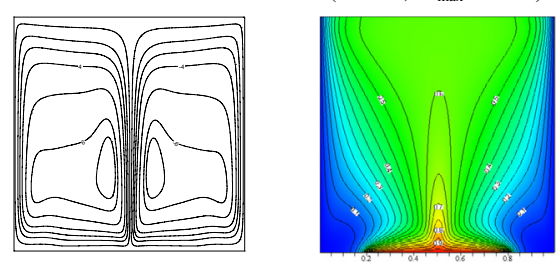

$\left(\mathrm{N}=30,\left|\Psi_{\text {max }}\right|=6.41\right)$

Isotherms
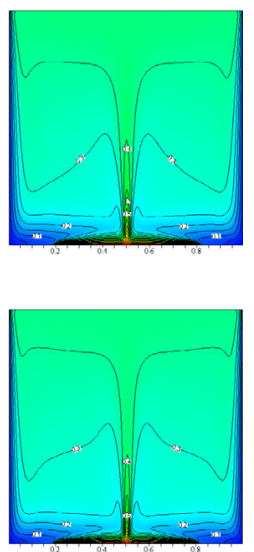

Iso-concentrations
Streamlines

Fig. 8. Impact of the buoyancy ratio, $L e=10, R a^{*}=100$, $W=0.60$.

ratio is still barely observed through the $\theta$ profiles, as the convection mechanism still less pronounced.

Figure 10 summarizes a series of predictions designed to document the impact of Lewis number and buoyancy ratio on the mass transfer rate. Through the latter, and for each value of Lewis number, the mean Sherwood number is found as an increasing function of the increase buoyancy ratio which can be linked to the flow intensity as reported previously (see Fig. 9). For a given $N$, the mass transfer still increases with Lewis number. Indeed, the Lewis number increases through the Schmidt number, since the Prandtl number is already fixed at 10 , what reduces the thickness of the solutal boundary layer and leads to important mass transfer.

About the heat transfer, Figure 10b, the previous description is shown to hold only for the buoyancy ratio. In other terms, the mean Nusselt number is also an increasing function of the latter $(N)$ as the $V$-velocity optimum continuously increases; what decreases the thickness of the corresponding boundary layer and leads to the primary-convective regime (see Fig. 9). With Lewis number all the opposite is observed, the heat transfer rate decreases as the thermal boundary layer thickness is a function of $L e^{1 / 2}[21]$.
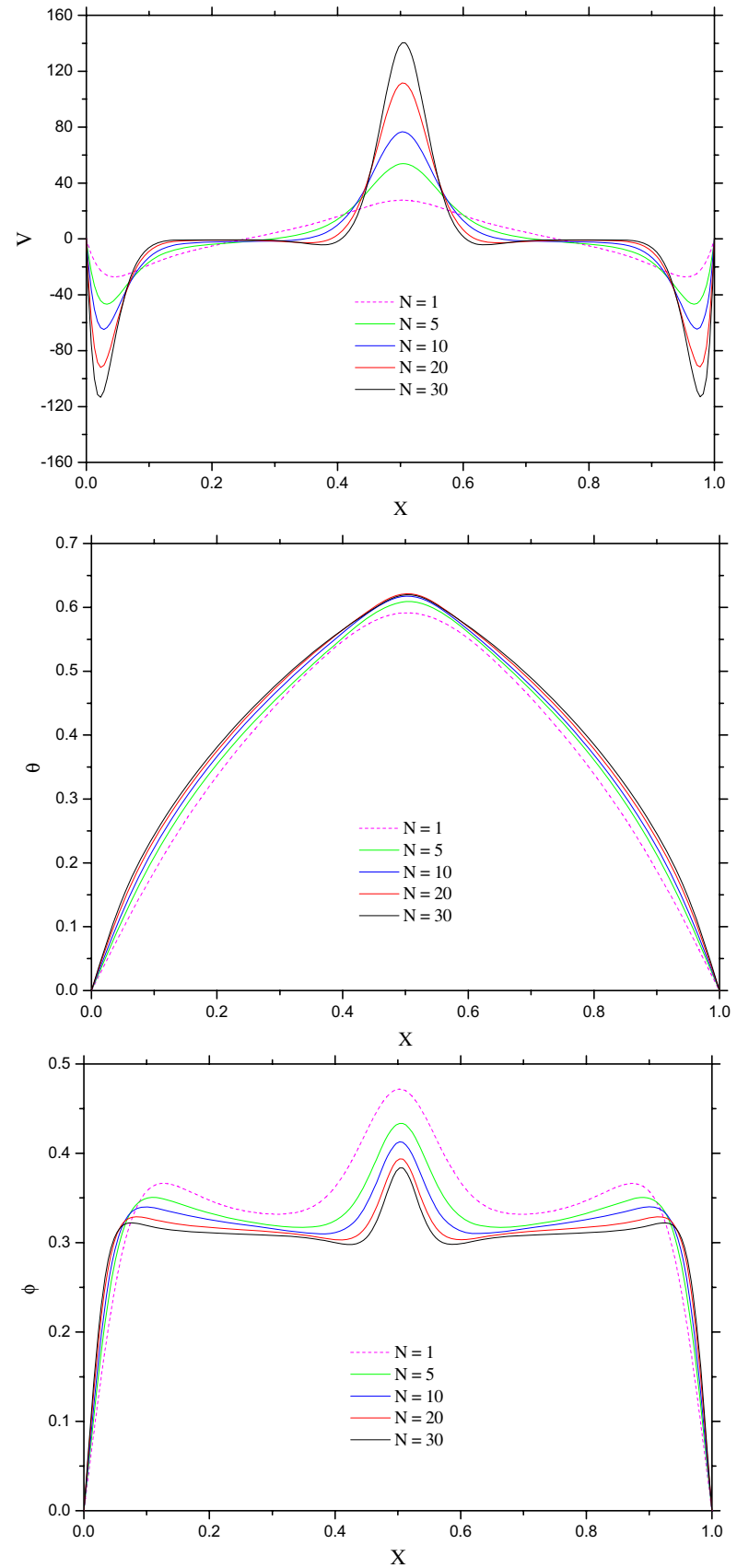

Fig. 9. $V$-Velocity, $\theta$ and $\phi$ profiles along the horizontal midplane of the porous square for various values of the buoyancy ratio. $L e=10, R a^{*}=100, W=0.60$.

\subsection{Impact of porous thermal Rayleigh number}

To complete the previous results, the evolution of heat and mass transfers with porous thermal Rayleigh number $\left(R a^{*}\right)$ is also discussed (Fig. 11). The Lewis number is taken as 10, which represents the case of hydrocarbon fuel [22], when the buoyancy ratio value is ranged between 0 and 30. As for the latter, the increase in the porous thermal Rayleigh number (through Grashof number) improves significantly the transfer rates, especially with high values of the buoyancy ratio. This behavior 

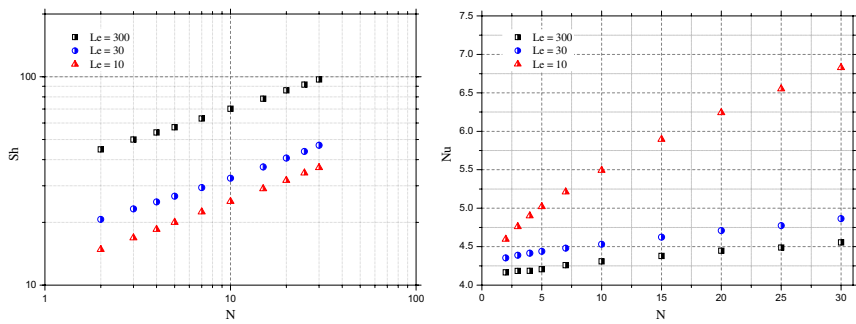

Fig. 10. Sherwood and Nusselt numbers as a function of the buoyancy ratio, for various values of Lewis number. $R a^{*}=$ $100, W=0.60$.
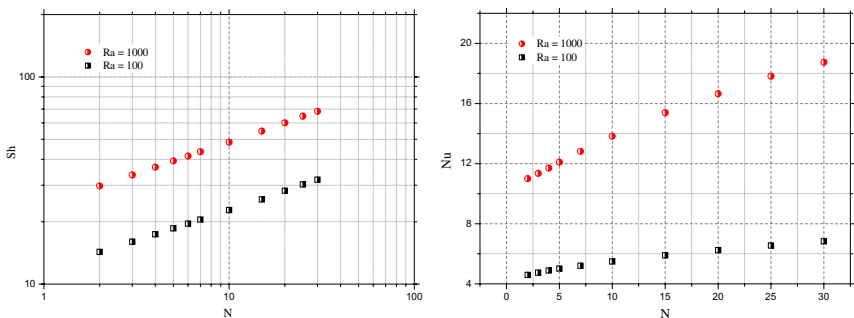

Fig. 11. Sherwood and Nusselt numbers as a function of the buoyancy ratio, for various values of the porous thermal Rayleigh number. $L e=10, W=0.60$.

confirms the fact that the global buoyancy term in the momentum equation, $\operatorname{Gr}_{T}(\theta+N \phi)$, increases with both; the porous thermal Rayleigh number $\left(D a G r_{T} P r\right)$ and the buoyancy ratio $(N)$, promotes consequently the flow intensity and so the overall transfer.

These primary observations allow us to conclude the proportional relation between the thermosolutal transfer, and the governing parameters such $L e, N$ and $R a^{*}$.

\subsection{Source length effect and proposed models}

For fixed values of Lewis number and buoyancy ratio, $(L e=N=10)$, the impact of the source length on the fluid dynamic, thermal and solutal behaviors into the porous square is displayed in Figure 12. As before, the two counter-rotating cells created by the fluid motion are formed inside the square. By increasing the source length the counter-rotating cells get stronger as the fluid motion is more pronounced. On the other hand, the development of thin thermal and solutal boundary layers over the active walls, as shown in Figures 12b and 12c, is a clear indication of important gradients near these surfaces, especially when the source length is about 0.80 .

For the entire range of governing parameters, and by taking into account the expression of the global buoyancy term in the momentum equation $G r_{T}(\theta+N \phi)$ our Nusselt and Sherwood predictions obtained for various values of the source length can be represented as a function of dimensionless groups such $R a^{*} N / L e$ and $R a^{*} L e(N+1)$ as shown in Figure 13. Through the latter, the proportional relation between the heat and mass transfers and the source length is well approved, what leads us eventu-
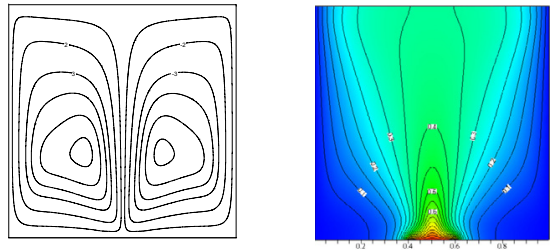

$\left(\mathrm{W}=0.20,\left|\Psi_{\max }\right|=3.88\right)$
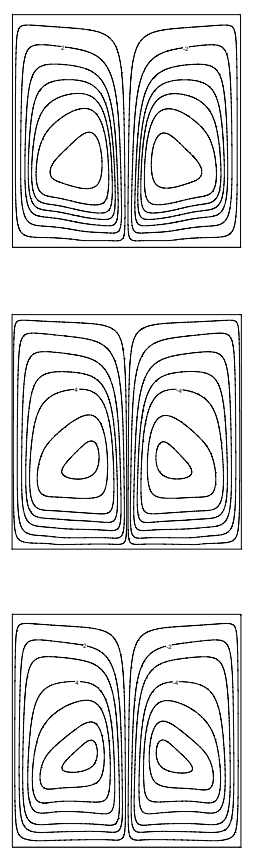

Streamlines

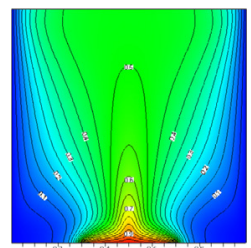

$\left(\mathrm{W}=0.40,\left|\Psi_{\max }\right|=4.42\right)$

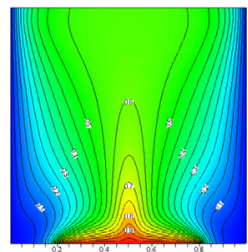

$\left(\mathrm{W}=0.60,\left|\Psi_{\max }\right|=4.76\right)$

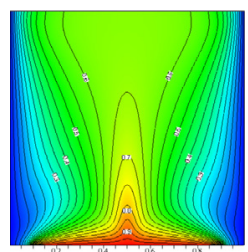

$\left(\mathrm{W}=0.80,\left|\Psi_{\max }\right|=5.56\right)$

Isotherms
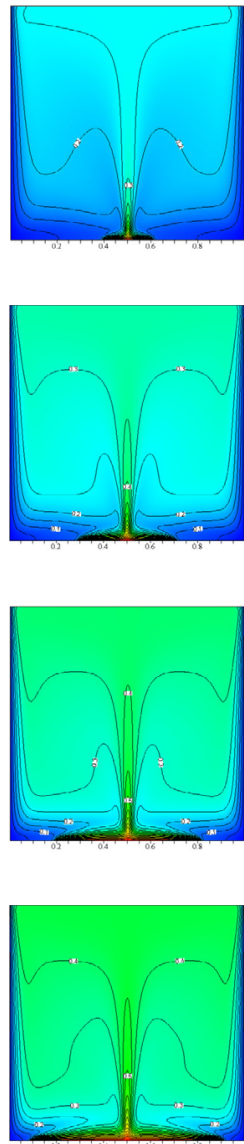

Iso-concentrations
Fig. 12. Impact of the source length. $L e=10, N=10$, $R a^{*}=100$.

ally to the following global correlations (Fig. 14):

$$
\begin{aligned}
& S h=0.788\left[\frac{R a * \operatorname{Le}(N+1)}{(1-W)^{1.2}}\right]^{0.32}\left(R^{2}=0.9940\right) \\
& N u=A+B \log \left[\left(\frac{R a^{*}|N|}{L e}(1-W)^{3}\right)^{0.48}\right] \\
& \quad+C\left\{\log \left[\left(\frac{R a^{*}|N|}{L e}(1-W)^{3}\right)^{0.48}\right]\right\}^{2}\left(R^{2}=0.9970\right)
\end{aligned}
$$

where the coefficients $A, B$ and $C$ of the Nusselt equation can be found based on the source length as follows:

$$
\left\{\begin{array}{l}
A=3.28-0.83 W+3.19 W^{2} \\
B=0.22-0.38 W+1.84 W^{2} \\
C=0.03-0.17 W+0.24 W^{2}
\end{array}\right.
$$

These powerful correlations, found to predict the numerical results within $\pm 1 \%$, may count as a complement to 

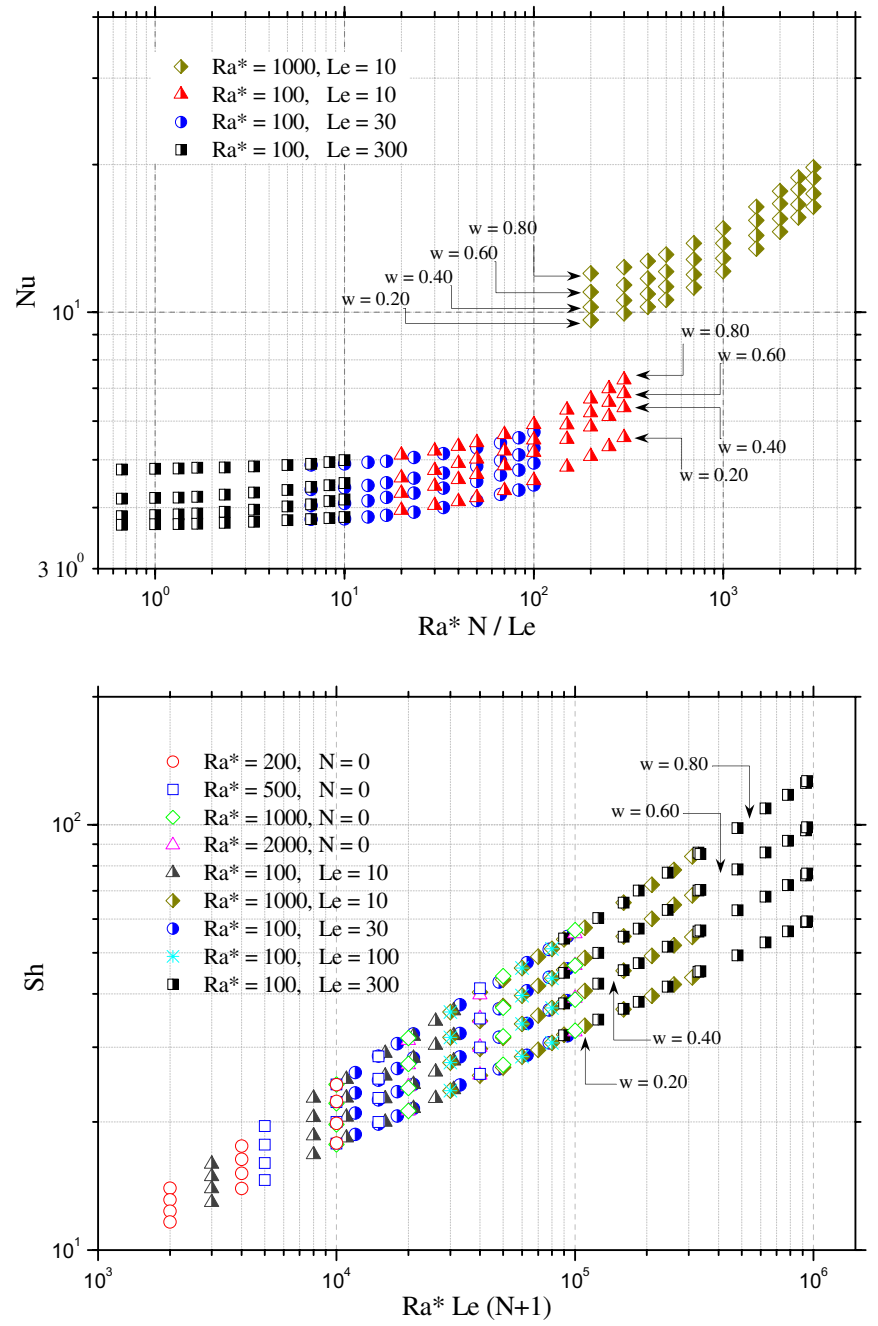

Fig. 13. Transfer rates as a function of dimensionless groups for various source length values.

previous researches done in the case of square porous enclosures saturated by a Newtonian fluid, and still available for a Lewis number ranging from 10 to 300, a buoyancy ratio taking between 0 and 50, a porous thermal Rayleigh number between 100 and 2000, and a source length between 0.10 and 0.90 .

\section{Conclusion}

The analysis of double-diffusive natural convection phenomenon inside a square Darcy-Brinkman porous enclosure having a bottom heat and solute source was realized numerically through this paper. By taking into account the effects of pertinent parameters such the buoyancy ratio, the source length, the Lewis and the porous thermal Rayleigh numbers, new powerful Sherwood and Nusselt correlations which display the heat and mass transfer rates in such geometry were proposed with an accuracy of about $1 \%$. These unique correlations may count as a complement to previous researches done into square
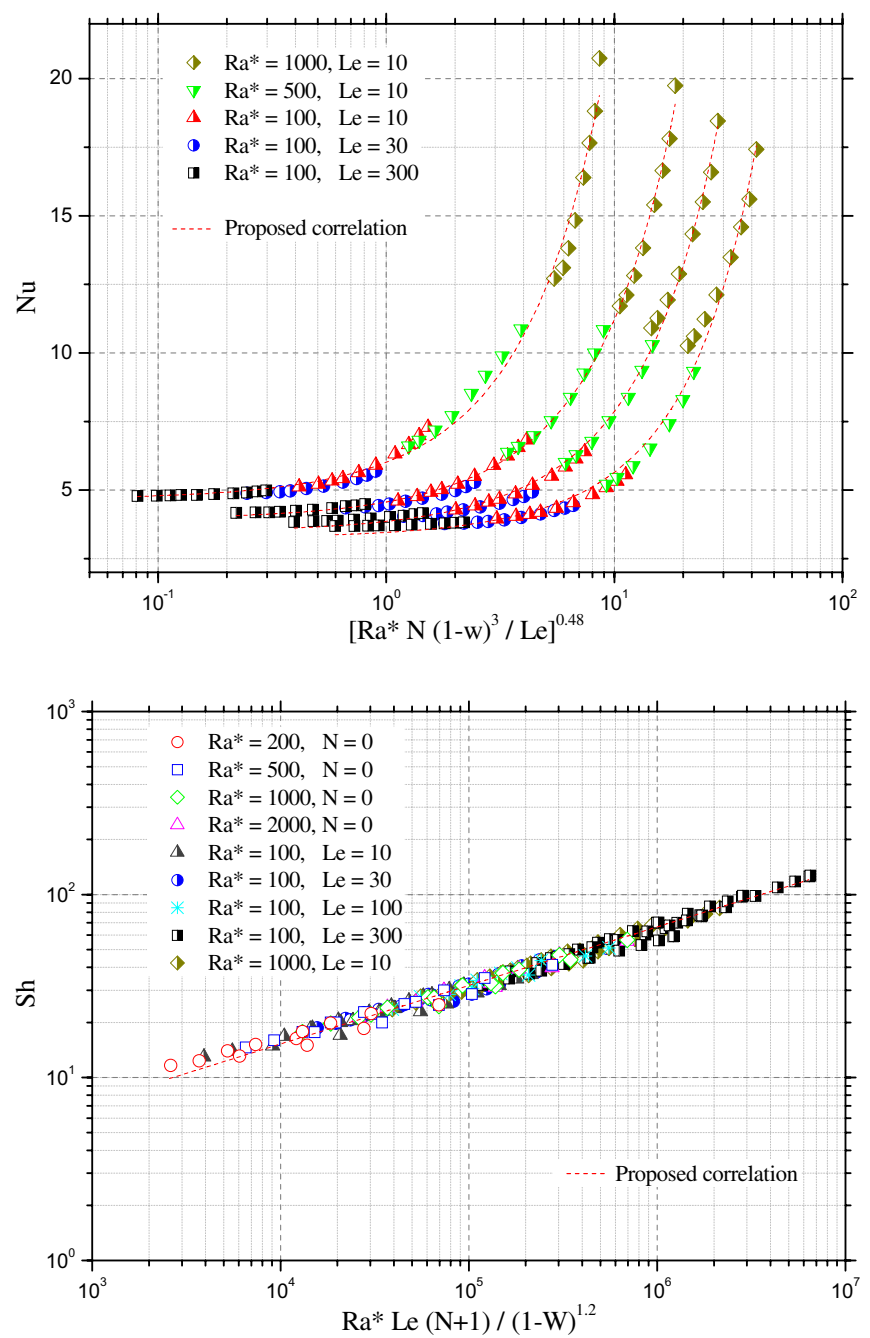

Fig. 14. Proposed numerical models for heat and mass transfer rates.

porous enclosures and might prove particularly useful in verifying any instability analysis which might be put forth in the future.

\section{References}

[1] D.A. Nield, A. Bejan, Convection in porous media, Springer, Berlin, 1992

[2] S. Ostrach, Natural convection with combined driving forces, Phys. Chem. Hydrodyn. 1 (1980) 233-247

[3] Y. Kamotani, L.W. Wang, S. Ostrach, H.D. Jiang, Experimental study of natural convection in shallow enclosures with horizontal temperature and concentration gradients, Int. J. Heat Mass Transfer 28 (1985) 165-173

[4] J. Lee, M.T. Hyun, K.W. Kim, Natural convection in confined fluids with combined horizontal temperature and concentration gradients, Int. J. Heat Mass Transfer 31 (1988) 1969-1977

[5] L.W. Wang, J.J. Chert, Flow patterns of thermosolutale convection in low aspect ratio enclosures, Experimental Heat Transfer 1 (1987) 197-204 
[6] M. Mamou, P. Vasseur, E. Belgen, Multiple solutions for double-diffusive convection in a vertical porous enclosure, Int. J. Heat Mass Transfer 38 (1995) 1787-1798

[7] C. Benard, D. Gobin, J. Thevenin, Thermosolutale natural convection in a rectangular enclosure: Numerical Results, in Heat Transfer in Convective Flows, edited by R.K. Shah, ASME, New York, 1989, pp. 249-254

[8] H. Han, T.H. Kuehn, A numerical simulation of double diffusive natural convection in a vertical rectangular enclosure, in Heat Transfer in Convective Flows, edited by R.K. Shah, ASME, New York, 1989, pp. 149-154

[9] J. Chang, T.F. Lin, C.H. Chien, Unsteady thermosolutal opposing convection of a liquid-water mixture in a square cavity - I: Flow formation and heat and mass transfer characteristics, Int. J. Heat Mass Transfer 36 (1993) 1315-1331

[10] J. Chang, T.F. Lin, Unsteady thermosolutal opposing convection of liquid-water mixture in a square cavity II: Flow structure and fluctuation analysis, Int. J. Heat Mass Transfer 36 (1993) 1333-1345

[11] D.A. Nield, Onset of thermohaline convection in a porous medium, Water Res. 4 (1968) 553-560

[12] J.W. Taunton, E.N. Lightfoot, T. Green, Thermohaline instability and salt fingers in porous medium, Phys. Fluids 15 (1972) 748-753

[13] D. Poulikakos, Double-diffusive convection in a horizontal sparsely packed porous layer, Int. Commun. Heat Mass Transfer 13 (1986) 587-598
[14] F. Chen, Double-diffusive fingering convection in a porous medium, Int. J. Heat Mass Transfer 36 (1993) 793-807

[15] O. Trevisan, A. Bejan, Heat and mass transfer by high Rayleigh number convection in a porous medium heated from below, Int. J. Heat Mass Transfer 30 (1987) 23412356

[16] T.F. Lin, C.C. Huang, T.S. Chang, Transient binary mixture natural convection in a square enclosure, Int. J. Heat Mass Transfer 33 (1990) 287-299

[17] R. Bennacer, Thermosolutal convection: fluid flow and heat transfer numerical simulations, Ph.D. thesis, Pierre and Marie Curie, Paris, 1993

[18] S.V. Patankar, Numerical heat transfer and fluid flow, Mc Grow, New York, 1980

[19] N. Hadidi, Y. Ould Amer, R. Bennacer, Bi-layered and inclined porous collector: Optimum heat and mass transfer, Energy 51 (2013) 422-430

[20] J.A. Weaver, R. Viskanta, Natural convection in binary gases driven by combined horizontal thermal and vertical solutal gradients, Exp. Thermal Fluid Sci. 5 (1992) 57-68

[21] O. Trevisan, A. Bejan, Natural convection with combined heat and mass transfer buoyancy effects in a porous medium, Int. J. Heat Mass Transfer 28 (1985) 1597-1611

[22] A.M. Abdulmajeed, R. Bennacer, Natural convection in a confined saturated porous medium with horizontal temperature and vertical solutal gradients, Int. J. Thermal Sci. 40 (2001) 82-93 